absolute eosinophil count of 4,000 per cu. $\mathrm{mm}$. or more ; thirdly, a positive filarial complement fixation test using Dirofilaria immitis as the antigen; and, lastly, a clinical response within two weeks to diethylcarbamazine. ${ }^{6}$ The syndrome is especially common in Indians wherever they live and particularly in the warm damp parts of the world. The evidence points to a non-human filarial infection as the cause, though possibly there is more than one aetiology. ${ }^{7}$

A similar clinical picture may be produced by visceral larva migrans, which was first described by P. C. Beaver and his colleagues in $1952 .^{8}$ This is a disease of children aged between one and three years who almost invariably have a history of pica. There is prolonged general ill health, and often the liver is enlarged. The filarial complement-fixation test is negative, though the fluorescent antibody test using larvae of Toxocara canis as the antigen is almost always positive. There is usually no improvement with carbamazine. The illness is probably caused by infestation with toxocara, whose natural host is the dog. Similar conditions may also be seen in the early invasive stage of schistosomal infections and in infections with Strongyloides stercoralis, but in neither of these are radiological changes found.

\section{Axillo-femoral Bypass}

To what length should a surgeon go to restore the circulation to a leg? This question is no longer hypothetical; the use of autogenous veins and synthetic prostheses has changed it to a literal one. Recently J. A. Mannick and D. C. Nabseth ${ }^{1}$ have reported 19 operations for the restoration of circulation to the leg by connecting the femoral artery to the axillary artery using a long, knitted Dacron tube. The patients were too ill to withstand transabdominal aorto-iliac surgery, but had gangrene or pain at rest and so were in need of relief. Three of the bypasses thrombosed, but the remaining 16 remained patent until the death of the patient or over the period of the follow-up-in one case 22 months.

This study confirms the work of J. H. Louw ${ }^{2}$ and F. W. Blaisdell and A. D. Hall ${ }^{3}$ that this operation is a relatively minor, simple procedure and also shows that valuable longterm successes can be achieved. The patient too ill to undergo abdominal surgery or with abdominal sepsis but with patent arteries below the groins is the ideal subject for this operation-for a good outflow tract will predispose towards success-but such patients are rare. Most patients who have pain at rest or gangrene have peripheral as well as aorto-iliac blocks. Mannick and Nabseth suggest that the presence of back-bleeding from the profunda femoris artery indicates an adequate outflow tract, but in practice even when technically successful aorto-iliac endarterectomies are done on patients fulfilling this criterion symptomatic relief is not invariable. The clinical problem is to decide which patients should have a long bypass graft and which should have an amputation. The vascular surgeon, quite rightly, views an amputation as a failure, but there are times when the correct amputation will get the patient walking much earlier than a distressing series of failed vascular operations. As the

\footnotetext{
' Mannick, J. A., and Nabseth, D. C., New Engl. F. Med., 1968, 278, 461.

261.
3 Bouw, J. H., Lancet, 1963, 1, 1401.
Blaisdell, F. W., and Hall, A. D., Surgery, 1963, 54, 563.
}

techniques and scope of peripheral vascular surgery increase the problem of the choice of operation grows. It is unfortunate that objective methods of preoperative assessment to guide the surgeon's decisions are not advancing at the same speed as his technical skill.

\section{Cardiovascular Surgery}

The European Society of Cardiovascular Surgery was formed in 1951 by the leading cardiac and peripheral vascular surgeons of Europe. Its seventeenth annual congress was held recently in London under the presidency of Professor J. B. Kinmonth.

The discussions on lymphoedema showed that much could be done to relieve patients with massive swollen legs by excisional operations or by using the buried dermis flap technique, but the innovation which aroused most interest was the use of lymphovenous shunts to reduce lymphoedema. Conflicting experimental evidence was presented on the effectiveness of such anastomoses between lymph nodes and veins, and no one produced any direct evidence of persisting patent shunts in man, but $J$. Nielubowicz (the originator of the method) presented a series of patients with secondary lymphoedema in which more than $50 \%$ were more mobile and had less pain and the majority had a reduction in limb volume.

The discussion on homograft heart valves was concerned mainly with the long-term results and the fate of biological tissues transplanted into the cardiovascular system. Most tissues are suitable in the short term, but many fatigue early. The effect of preparation and sterilization on the structure of homologous and heterologous valves was discussed in detail. Some of the best long-term results have been obtained with homologous, sterilized, but not freeze-dried, valves, though all types are liable to stiffening and calcification as they get older. The general opinion was that the homograft and the autograft were the valves to be used and studied in the next few years.

The third symposium considered the results of surgery on the main branches of the aortic arch. Obstruction of these vessels causes a variety of cerebral ischaemic syndromes, and the results of endarterectomy or bypass operations appear to be good provided the cerebral symptoms are transient and there is no gross generalized disease. It is important that the general physician should be aware of these syndromes so that the patient can reach the surgeon before he has permanent neurological damage.

The symposium on multivalvar cardiac lesions reflected the great advances of surgical technique in this field and the amazingly good results that can be obtained by replacing three diseased valves. The mortality rate is still high but below $25 \%$ in good centres. It might be said that it is easier to transplant a new heart than repair three valves. Cardiac transplantation was not on the programme, but in view of current interest and the presence at the meeting of representatives from the Paris, Houston, and London transplant teams special arrangements were made for them to present accounts of the clinical details of their work and their opinions. This conference displayed, more than most, the inestimable value for doctors of being able to sit down and talk privately with their colleagues. 